\title{
Lower androgen levels promote abnormal cartilage development in female patients with adolescent idiopathic scoliosis
}

\author{
Yuan-Tao Wu ${ }^{1,2,3}$, Ming-Xing Tang ${ }^{1,2}$, Yun-Jia Wang ${ }^{1,2}$, Jiong $\mathrm{Li}^{1,2}$, Yu-Xiang Wang ${ }^{1,2}$, Ang Deng ${ }^{1,2}$, \\ Chao-Feng Guo ${ }^{1,2}$, Hong-Qi Zhang ${ }^{1,2}$
}

${ }^{1}$ Department of Spine Surgery and Orthopaedics, Xiangya Hospital, Central South University, Changsha, China; ${ }^{2}$ National Clinical Research Center for Geriatric Disorders, Xiangya Hospital, Central South University, Changsha, China; ${ }^{3}$ Department of Spine Surgery, Hainan General Hospital and Hainan Affiliated Hospital of Hainan Medical University, Haikou, China

Contributions: (I) Conception and design: YT Wu; (II) Administrative support: CF Guo, HQ Zhang; (III) Provision of study materials or patients: HQ Zhang, J Li; (IV) Collection and assembly of data: MX Tang, YX Wang; (V) Data analysis and interpretation: YJ Wang, A Deng; (VI) Manuscript writing: All authors; (VII) Final approval of the manuscript: All authors.

Correspondence to: Hong-Qi Zhang; Chao-Feng Guo. Department of Spine Surgery and Orthopaedics, Xiangya Hospital, Central South University, Changsha 410008, China. Email: zhq9996@163.com; guochaofeng2016@126.com.

Background: Adolescent idiopathic scoliosis (AIS) is a disease characterized by changes in the threedimensional structure of the spine. Studies have shown that the development of AIS might be associated with genetic, biomechanics, endocrine factors and abnormal bone or cartilage development.

Methods: Blood samples collected from 301 female patients (161 females with AIS and 140 females without AIS) were used for genotyping. Forty-eight serum samples from 161 females with AIS and 40 serum samples from 140 females without AIS were subjected to enzyme-linked immunosorbent assays (ELISAs). We also evaluated 32 facet joints (18 females with AIS and 14 females without AIS from the 301 female patients) using immunohistochemistry, Western blotting, and isolation of human primary chondrocytes, among other methods. We treated the AIS primary chondrocytes with dihydrotestosterone (DHT) to verify the relationship among androgen, the androgen receptor (AR), and its downstream pathway proteins.

Results: The serum androgen level in the AIS group was significantly decreased $(1.94 \pm 0.09$ vs. $2.284 \pm 0.103)$ compared with that in the non-AIS (control) group. The single nucleotide polymorphism genotyping results showed that the mutation rates of rs6259 between the AIS and control groups were significantly different (G/G genotype: $48.4 \%$ vs. $42.1 \%$, G/A genotype: $40.4 \%$ vs. $35.7 \%, \mathrm{P}<0.05$ ). The levels of interleukin (IL)6 and metalloproteinase (MMP)-13 were increased in the cartilage of AIS patients, and these patients also exhibited decreased AR levels. The cell experiment results showed that androgen reduced the degree of abnormal cartilage development in female AIS patients through the AR/IL-6/signal transducer and activator of transcription 3 (STAT3) signaling pathway.

Conclusions: Our study provides a new perspective on the pathogenesis of AIS and indicates that decreased androgen levels in female AIS patients play a potential role in the development of AIS via the AR/ IL-6/STAT3 signaling pathway.

Keywords: Adolescent idiopathic scoliosis (AIS); androgen; interleukin (IL)-6; signal transducer and activator of transcription 3 (STAT3); cartilage

Submitted Apr 06, 2020. Accepted for publication Jan 25, 2021.

doi: 10.21037/atm-20-3171

View this article at: http://dx.doi.org/10.21037/atm-20-3171 


\section{Introduction}

Adolescent idiopathic scoliosis (AIS) is a disease characterized by changes in the three-dimensional structure of the spine. According to epidemiological statistics, the prevalence of scoliosis is between $1 \%$ and $4 \%$ (1). The pathogenesis of AIS remains unclear, but recent studies have shown that the development of AIS might be associated with genetics (2), hormone and endocrine factors, and biomechanics (3). Many scholars have shown that patients with AIS abnormally secrete many types of hormones, such as estrogen, leptin, and ghrelin (4-6), and changes in the levels of these hormones can induce abnormal bone and cartilage development and might eventually result in AIS. These results suggest that hormone abnormalities might be an important pathogenic factor in the development of AIS.

Clinical presentations of AIS are not evident in childhood. The disease usually develops in adolescence, and many patients experience rapid disease progression. Specifically, the secretion levels of various hormones increase rapidly during adolescence, AIS is more common in females, and the overall ratio of girls to boys with scoliosis is 11:1 (7), indicating that sex hormones might be key factors for the pathogenesis and progression of AIS caused by hormone abnormalities. Many studies have demonstrated that estrogen can promote the development of AIS $(4,8)$. However, few studies are available on the function of androgen in the pathogenesis of AIS. The term androgen is derived from the ancient Greek word "andro", and androgen is usually defined as a male hormone that is secreted by testicular interstitial cells; however, the adrenal cortex and female ovary can also synthesize a small amount of androgen (9). Because the organs that secrete androgen in males and females are different and the individual contents show wide variations, direct comparisons are not suitable. Furthermore, this disease mainly occurs in female; therefore, only female patients were used as participants in this study. Serum samples from 88 adolescent female patients (48 females with AIS and 40 females without AIS) were subjected to enzyme-linked immunosorbent assays (ELISAs), and the results showed that androgen levels in patients with AIS were decreased compared with those in females without AIS (control), indicating that serum androgen levels might be associated with AIS. Recent studies confirmed that androgen is closely associated with bone metabolism, skeletal muscle growth, and protein synthesis and metabolism. Almeida et al. showed that androgen is involved in trabecular bone maintenance and cortical bone growth (10). The androgen receptor (AR) is extensively distributed throughout the body, and in bone tissue, the AR is mainly distributed in growth sites where proliferation and maturation are promoted during endochondral ossification and in bone remodeling sites, demonstrating that androgen directly acts on cartilage and bone (11). A study conducted by Cicuttini $e t$ al. indicated that androgen is positively correlated with the male tibial cartilage volume (12). In addition, a study performed by Steffens et al. showed that androgen regulates experimental bone loss through the AR (13). Although androgen is closely associated with bone development, the role of androgen in the development of AIS and whether androgen induces abnormal cartilage or bone development in patients with AIS have not been reported. Furthermore, serum androgen levels are affected by many factors. Recent studies confirmed that single nucleotide polymorphism (SNP) loci, including rs12150660, rs727428, rs6259, rs5934505, rs10822184, and rs6258, are associated with serum androgen levels (14-16). Therefore, we enrolled 161 females with AIS and 140 females without AIS and performed genotyping to determine the causes of the reductions in the androgen level observed in patients with AIS.

Our previous studies showed that interleukin (IL)6 expression is increased in the cartilage of patients with AIS, suggesting that IL-6 might be associated with the development of AIS. A study conducted by Cho DC et al. showed that androgen blocks IL- 6 to promote cortical bone formation in mice (17), indicating that IL-6 expression in patients with AIS might be correlated with androgen abnormalities. In addition, numerous studies have indicated that IL-6 is closely associated with cartilage abnormalities in patients with osteoarthritis (18-20). As a member of the interleukin family, IL-6 can be secreted by many types of cells and can induce the proliferation and differentiation of many cell types. A study conducted by Yamaguchi et al. showed that chondrocytes in patients with hip synovitis secrete IL-6 and stimulate synovial cell proliferation (21). Previous studies have confirmed that IL- 6 promotes the differentiation of mesenchymal stem cells into chondrocytes and the self-repair of cartilage through the IL-6/STAT3 pathway (22). However, whether IL-6 is associated with the development and progression of AIS has not been reported. Therefore, this study investigated whether androgen and IL-6 abnormalities induce abnormal cartilage development in patients with AIS. 
Table 1 Clinical data of the enrolled female AIS patients

\begin{tabular}{lccc}
\hline Items & AIS & Control & P value \\
\hline Number & 161 & 140 & - \\
Age & $12.6 \pm 3.5$ & $11.2 \pm 4.2$ & 0.15 \\
Main curve cobb angle & $37.5 \pm 12.4$ & - & - \\
\hline $\begin{array}{l}\text { Student's } t \text {-test was used for the statistical analysis, and a P } \\
\text { value }>0.05 \text { indicated no significant difference between the two } \\
\text { groups. }\end{array}$
\end{tabular}

\section{Methods}

\section{Subjects}

All of the specimens were collected from female AIS patients between 10 and 17 years of age and matched patients without AIS (Table 1). The criterion used for selection of the AIS patients was a minimum Cobb angle greater than 10, and a clinical assessment was conducted every 3 months. Patients with a congenital spinal deformity, Marfan syndrome, neuromuscular diseases, or spinal deformities caused by other syndromes were excluded from this research. The inclusion criteria for the control group were age-matched non-AIS patients, including healthy individuals and patients with lumbar herniation or spine fracture at our medical examination center. The patients in the control group were subjected to a comprehensive $\mathrm{X}$-ray examination to preclude any spinal deformity.

This study was approved by the Ethics Committee of Xiangya Hospital of Central South University (No. 201703358). Written informed consent was provided by all the subjects and their legal guardians prior to participation in the study, and the study was performed in accordance with the tenets of the Declaration of Helsinki (as revised in 2013) and its amendments.

\section{Isolation and culture of human primary chondrocytes}

We collected 32 facet joints from 18 AIS patients and 14 patients without AIS during spinal surgery; the enrolled non-AIS patients were generally patients with spinal fracture or lumbar disc herniation who needed surgery. All of the cartilage was peeled from the facet joints, cut into slices with a thickness of approximately $0.5 \mathrm{~mm}$, washed with phosphate-buffered saline (PBS), and treated with $0.25 \%$ trypsin (Gibco, USA) for $30 \mathrm{~min}$ and $1 \mathrm{ng} / \mathrm{mL}$ collagenase type II (Sigma-Aldrich, USA) for 4 h at $37^{\circ} \mathrm{C}$. The fragment was then plated into 6 - $\mathrm{cm}$ culture dishes.
All chondrocytes were cultured in DMEM/high-glucose medium (Gibco, USA) supplemented with 10\% fetal bovine serum (Gibco, USA) and $1 \%$ penicillin/streptomycin (Gibco, USA) at $37{ }^{\circ} \mathrm{C}$ in an incubator with $5 \% \mathrm{CO}_{2}$. All the cells were passaged twice before use.

\section{Enzyme-linked immunosorbent assay (ELISA)}

The serum androgen level and the secretion of IL-6 from chondrocytes into the cell culture medium were measured using a commercial ELISA kit (Cusabio Biotech, China) in accordance with the manufacturer's recommended protocols.

\section{Genotyping}

We used the SQ blood DNA Kit II (Omega Bio-TEK, USA) to extract genomic DNA from peripheral blood, and SNP genotyping was performed using the improved multilink detection reaction (iMLDR) technique developed by Genesky Biotechnology Company (China). Different allelespecific oligonucleotide probes were used to identify the alleles for each SNP, and different extension lengths at the 3' end were used to further distinguish different SNPs. We set up two negative controls, namely, double-distilled water as the template and a DNA sample without primers, while keeping the other conditions unchanged. Random samples accounting for $5 \%$ of the total DNA samples were sequenced using Big Dye-Terminator version 3.1 and an ABI 3730XL automatic sequencer (a biological application system) to confirm the iMLDR results.

\section{Cell proliferation assay}

After intervention, chondrocytes were seeded into 96-well plates at a density of 3,000 cells per well. At 12, 24, 48, and $72 \mathrm{~h}$ after seeding, cell viability was measured using the Cell Counting Kit-8 (CCK-8) system (Dojindo, Japan) according to the manufacturer's instructions. The results were detected at $450 \mathrm{~nm}$.

\section{siRNA transfection}

First, the cells were seeded into 24-well plates at a density of $1 \times 10^{4}$ cells per well. The cells were then transfected with siRNAs against $\mathrm{AR}$ using riboFECT ${ }^{\mathrm{TM}} \mathrm{CP}$ reagent at final siRNA concentrations of $50 \mathrm{nM}$ following the manufacturer's recommended protocols. The siRNA 
sequences used to silence AR expression are shown in Table S1. Forty-eight hours after transfection, the expression level of AR protein was verified by fluorescence microscopy and Western blotting (WB).

\section{Real-time quantitative PCR}

The experimental method used for qRT-PCR was based on a previously described method (18). The following primers were used in this study: $18 \mathrm{~S}$, CCCTCCAATGGATCCTCGTT (forward) and AGAAACGGCTACCACATCCA (reverse); IL6, AgGAGACTTGCCTGGTGAAA (forward) and CAGGGGTGGTTATTGCATCT (reverse); and AR, GACGCTTCTACCAGCTCACC (forward) and GCTTCACTGGGTGTGGAAAT (reverse).

\section{Western blotting}

We collected total protein from chondrocytes using RIPA lysis buffer (CWBIO, China) containing phosphatase inhibitor and PMSF, and the protein was then denatured in a metal bath thermostat. The proteins were separated by $10 \%$ SDS-PAGE and then transferred to PVDF membranes (Bio-Rad, Hercules, USA). The membranes were subsequently blocked with $5 \%$ skim milk for $1 \mathrm{~h}$ and incubated overnight with primary antibodies against GAPDH $(1: 2,000$, \# 5174S, RRID:AB_10622025, Cell Signaling Technology, CST, USA), AR (1:200, ab198394, RRID:AB_2861275, Abcam, USA), IL-6 (1:800, ab6672, RRID:AB_2127460, Abcam), metalloproteinase (MMP)13 (MMP13) (1:800, \# 94808, RRID:AB_2800235, CST), STAT3 (1:800, \# 12640S, RRID:AB_2629499, CST), and p-STAT3 (1:500, \# 9134S, RRID:AB_331589, CST). The membranes were then incubated with secondary antibodies $(1: 8,000)$ at room temperature for $1 \mathrm{~h}$. The results were then detected using the Chemiluminescent Protein Detection Module (Thermo Scientific, USA).

\section{Immunochemistry (IHC)}

The tissues collected during surgery were fixed with formalin for $24 \mathrm{~h}$ and decalcified in 10\% EDTA for 15 days. The tissues were cut into 5-mm-thick slices, and the paraffinized sections were treated with xylene and rehydrated with a graded alcohol series $(100 \% ; 95 \% \times 2$, $85 \% \times 2$, and $70 \%$ for $3 \mathrm{~min}$ each) and double-distilled water for $5 \mathrm{~min}$. The slices were then pretreated with $3 \%$ hydrogen peroxide for $10 \mathrm{~min}$, blocked with $5 \%$ bovine serum albumin (BSA) for $30 \mathrm{~min}$, and washed with PBS. The sections were incubated with primary antibodies against AR (1:200, ab198394, RRID:AB_2861275, Abcam) and IL-6 (1:800, ab6672, RRID:AB_2127460, Abcam) overnight at $4{ }^{\circ} \mathrm{C}$ and subsequently with biotinylated goat anti-rabbit immunoglobulins for $30 \mathrm{~min}$ and a streptavidinhorseradish peroxidase solution (ZSGBBIO, China) containing the secondary antibody for $45 \mathrm{~min}$. The sections were then stained with 3,30-diaminobenzidine (DAB) for $30 \mathrm{~s}$ and counterstained with hematoxylin (Servicebio, China) for $1 \mathrm{~min}$. Pictures were acquired via using a microscope (Leica, Germany). The results were detected quantitatively via using ImageJ software (Bethesda, USA).

\section{Immunofluorescent staining}

AIS primary chondrocytes after incubation with IL-6 were first washed with PBS, fixed with $4 \%$ paraformaldehyde for $25 \mathrm{~min}$, incubated with $0.5 \%$ Triton X-100 for $10 \mathrm{~min}$, and then blocked with $5 \%$ BSA in PBS for $30 \mathrm{~min}$. Chondrocytes were then incubated overnight with primary antibody against Ki67 (1:500; \# 9449; RRID: AB_2797703; CST) at $4{ }^{\circ} \mathrm{C}$. After washing with PBS, the chondrocytes were incubated with goat anti-rabbit secondary antibody and Alexa Fluor 488-conjugated antibody (1:300; Sigma, USA) for $1 \mathrm{~h}$. Finally, the cells were incubated with DAPI (Solarbio, China) for $5 \mathrm{~min}$. Pictures were acquired by immunofluorescence microscopy (Leica, Germany). The results were detected quantitatively using ImageJ software (Bethesda, USA).

\section{Statistics}

The data obtained in the genetic association study were analyzed by the Chi-square (and Fisher's exact) test. Oneway ANOVA and Student's $t$-test were used to analyze the remaining data, and the quantitative data are expressed as the means \pm standard deviations (SDs). All analyses were performed using GraphPad Prism software (GraphPad Prism Software, USA), and $\mathrm{P}$ values $<0.05$ indicated statistical significance.

\section{Results}

Serum levels of androgen in females with AIS and females without AIS

To detect the serum level of androgen in AIS patients, we 


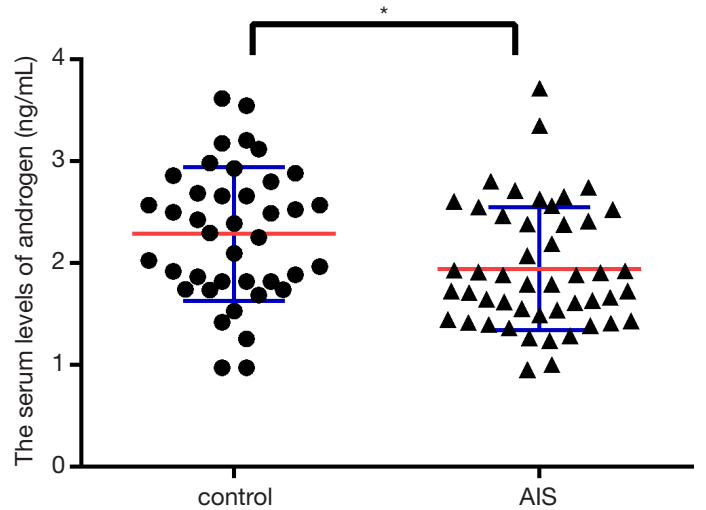

Figure 1 Serum levels of androgen in female AIS and nonAIS patients. The serum androgen level in the AIS group was lower than that in the control group $(1.94 \pm 0.09$ vs. $2.284 \pm 0.103)$. *indicates a significant difference compared with the control group $(\mathrm{P}<0.05)$.

analyzed serum samples from 48 females with AIS and 40 age-matched females without AIS by ELISA. The clinical information for all the patients is provided in Table 1. No differences in age or body weight were found between the AIS and control groups. The ELISA results showed that the serum androgen level in the AIS group was lower than that in the control group $(1.94 \pm 0.09$ vs. $2.284 \pm 0.103)$ (Figure 1).

\section{SNP genotype frequency distributions in AIS and non-AIS patients}

We genotyped eight SNPs that were previously shown to be related to the serum androgen levels. Specifically, a total of 161 AIS patients and 140 controls were successfully genotyped and subjected to statistical analysis. The eight genotyped SNPs in the two groups were analyzed using the Chi-square (and Fisher's exact) test. The genotype distribution frequencies are listed in Table 2.

A significant difference in the presence of rs6259 was detected between the two groups $(\mathrm{P}<0.05)$. Overall, the frequencies of the G/G and G/A genotypes in the AIS group were significantly higher than those in the non-AIS group (G/G genotype: $48.4 \%$ vs. $42.1 \%$, G/A genotype: $40.4 \%$ vs. $35.7 \%$, and $\mathrm{A} / \mathrm{A}$ genotype: $11.2 \%$ vs. $22.2 \%$; $\mathrm{P}<0.05)$.

\section{Increased IL-6 expression and reduced AR expression in the cartilage of AIS patients}

To confirm that androgen expression is associated with cytokines and proteins in the cartilage tissue of patients with AIS, the cartilage tissue separated from the facet joint tissue of the patients during surgery was analyzed by WB and IHC. WB analysis of total proteins extracted from the tissues showed that the cartilage of patients with AIS exhibited higher protein expression levels of IL-6 and MMP-13 and a lower protein expression level of the AR compared with the cartilage of non-AIS patients (Figure $2 A, B$ ). In addition, the IHC results showed that patients with AIS exhibited increased IL-6 expression and decreased AR expression in cartilage compared with the control group (Figure 2C,D,E,F).

\section{Androgen inbibits the secretion of IL-6 in AIS primary chondrocytes}

To confirm the effect of androgen on primary chondrocytes from patients with AIS, AIS primary chondrocytes were treated with $50 \mathrm{nM}$ dihydrotestosterone (DHT), whereas the control group was treated with PBS. After $48 \mathrm{~h}$ of treatment, the culture medium from the chondrocytes was collected for ELISA, and the results showed that the IL-6 level in the culture medium after DHT treatment was decreased compared with that in the control group, indicating that androgen inhibited the secretion of IL-6 from chondrocytes (Figure 3A). Total RNA and protein were extracted from AIS primary chondrocytes. PCR analysis showed that the mRNA level of IL-6 was decreased (Figure $3 B, C$ ), and $\mathrm{WB}$ revealed a decreased IL-6 protein level and increased AR expression (Figure $3 D, E, F$ ). Furthermore, the expression of MMP13 did not significantly change after androgen treatment (Figures S1,S2).

\section{The function of androgen in blocking IL-6 expression requires AR activation}

We further investigated the mechanism underlying the regulation of IL-6 expression by androgen. Therefore, we transfected AIS primary chondrocytes with siAR or siNC for $48 \mathrm{~h}$ and then treated the chondrocytes with $50 \mathrm{nM}$ DHT or PBS (control group). The siRNA transfection efficiency was verified by fluorescence microscopy and WB (Figures S3,S4). After treatment, total RNA and protein were collected, and WB analysis showed that IL-6 expression in the DHT + siAR group was significantly increased compared with that in the DHT + siNC group and was significantly different from that in the control group (Figure $4 A, B$ ). In addition, although the mRNA level 
Table 2 Frequency distributions of allele SNPs

\begin{tabular}{|c|c|c|c|}
\hline Single nucleotide polymorphism & Group AIS number (\%) & Group control number (\%) & $P$ value (AIS vs. control) \\
\hline $\mathrm{G} / \mathrm{G}$ & $78(48.4)$ & $59(42.1)$ & 0.03 \\
\hline $\mathrm{G} / \mathrm{A}$ & $65(40.4)$ & $50(35.7)$ & \\
\hline $\mathrm{A} / \mathrm{A}$ & $18(11.2)$ & $31(22.2)$ & \\
\hline $\mathrm{C} / \mathrm{C}$ & $20(12.4)$ & $19(13.6)$ & 0.85 \\
\hline $\mathrm{C} / \mathrm{T}$ & $88(54.7)$ & $72(51.4)$ & \\
\hline $\mathrm{T} / \mathrm{T}$ & $53(32.9)$ & $49(35.0)$ & \\
\hline \multicolumn{4}{|l|}{ rs12150660 (SHBG) } \\
\hline$A / A$ & $25(15.5)$ & $22(15.7)$ & \\
\hline \multicolumn{4}{|l|}{ rs11642015 (FTO) } \\
\hline $\mathrm{C} / \mathrm{C}$ & $121(75.2)$ & $101(72.2)$ & 0.48 \\
\hline $\mathrm{C} / \mathrm{T}$ & $39(24.2)$ & $36(25.7)$ & \\
\hline $\mathrm{T} / \mathrm{T}$ & $1(0.6)$ & $3(2.1)$ & \\
\hline \multicolumn{4}{|l|}{ rs266719 (EIF4A2) } \\
\hline $\mathrm{C} / \mathrm{C}$ & $133(82.6)$ & $112(80.0)$ & 0.51 \\
\hline $\mathrm{C} / \mathrm{T}$ & $28(17.4)$ & 27 (19.3) & \\
\hline $\mathrm{T} / \mathrm{T}$ & $101(62.8)$ & $90(64.3)$ & \\
\hline \multicolumn{4}{|l|}{ rs7639352 (FTO) } \\
\hline $\mathrm{C} / \mathrm{C}$ & $75(46.6)$ & $73(52.1)$ & 0.62 \\
\hline $\mathrm{C} / \mathrm{T}$ & $75(46.6)$ & $59(42.2)$ & \\
\hline$T / T$ & $11(6.8)$ & $8(5.7)$ & \\
\hline \multicolumn{4}{|l|}{ rs1799941 (SHBG) } \\
\hline $\mathrm{G} / \mathrm{G}$ & $160(99.3)$ & $140(100.0)$ & 1 \\
\hline $\mathrm{G} / \mathrm{A}$ & $1(0.7)$ & 0 & \\
\hline $\mathrm{A} / \mathrm{A}$ & 0 & 0 & \\
\hline
\end{tabular}


A

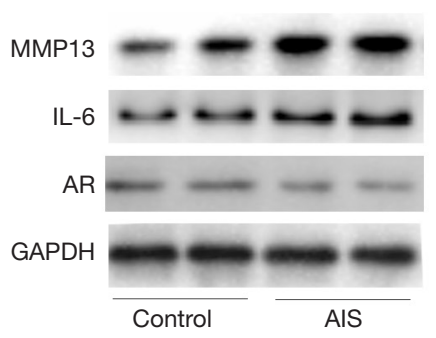

B

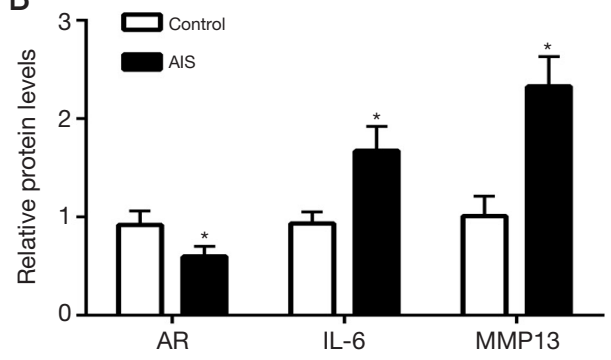

AIS

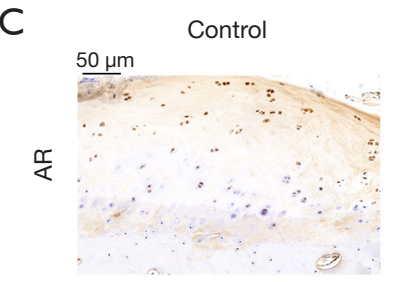

E

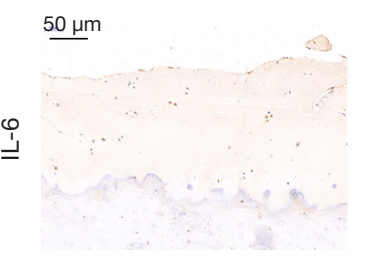

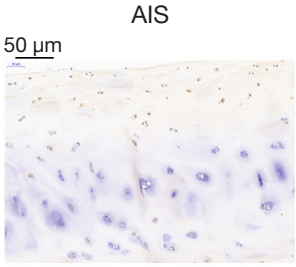

$50 \mu \mathrm{m}$

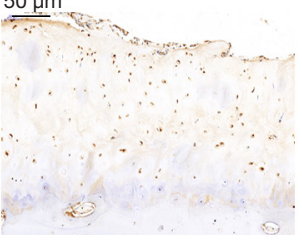

D

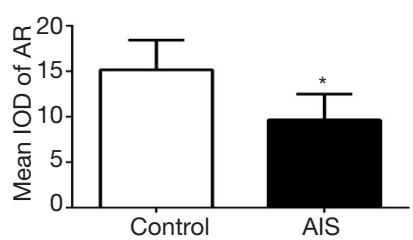

$\mathrm{F}$

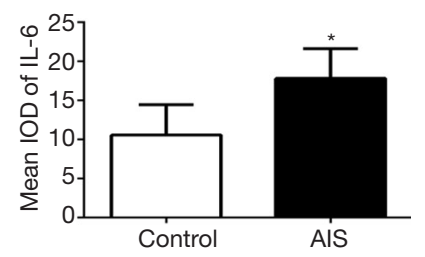

Figure 2 Increased IL-6 expression and reduced AR expression in the cartilage of AIS patients. (A,B) The protein levels of AR, IL-6, and MMP13 in the cartilage of female AIS and non-AIS patients. (C,D,E,F) AR and IL-6 levels detected by IHC in the cartilage of AIS and nonAIS patients; scale bar: $50 \mu \mathrm{m}$. The data are shown as the means \pm SDs. *indicates $\mathrm{P}<0.05$ vs. the control group.

of IL-6 in the siAR-transfected group was not significantly different from that in the siNC group, its expression level was higher than that in the siNC group (Figure 4C). These results indicate that the function of androgen in blocking IL-6 expression requires AR activation.

\section{Promotion of MMP13 expression and inbibition of chondrocyte proliferation by IL-6}

To study the effect of IL-6 overexpression on cartilage tissue, primary chondrocytes from patients with AIS were directly treated with $20 \mathrm{nM}$ IL-6 or PBS (control group). Total protein from the chondrocytes was collected for WB detection, and the results showed that the protein expression of MMP13 in chondrocytes was significantly increased after IL-6 treatment (Figure 5A,B). In addition, chondrocyte proliferation was assessed with Ki67 12, 24, 48, and $72 \mathrm{~h}$ after IL-6 treatment using the CCK- 8 assay, and the results showed that the proliferation ability of chondrocytes from AIS patients decreased after treating with $20 \mathrm{nM}$ IL-6 for $24 \mathrm{~h}$ (Figure 5C). Furthermore, the proliferation of primary cells from both the AIS and non-AIS groups was assessed. Interestingly, the proliferation rate obtained for the AIS group was slightly slower than that found for the nonAIS group, but the results were not significantly different (Figure 5D). Ki67 immunofluorescence staining was also used to determine AIS chondrocyte proliferation after IL-6 intervention and the chondrocytes proliferation in cartilage from AIS and non-AIS patients. The results showed that IL-6 reduced the proliferation ability of chondrocytes (Figure 5E,F), but tissue staining results showed that there was no significant difference in chondrocytes proliferation between AIS patients and non-AIS patients (Figure S5).

\section{Effects of IL-6 on chondrocytes from AIS patients mediated by STAT3 signaling pathway proteins}

Our results showed that the phosphorylation level of STAT3 protein in chondrocytes from AIS patients was higher than that in chondrocytes from non-AIS patients (Figure 6A,B). STAT3 is the most specific downstream protein in the IL-6 pathway. To confirm that the effect of IL-6 on chondrocytes 
A

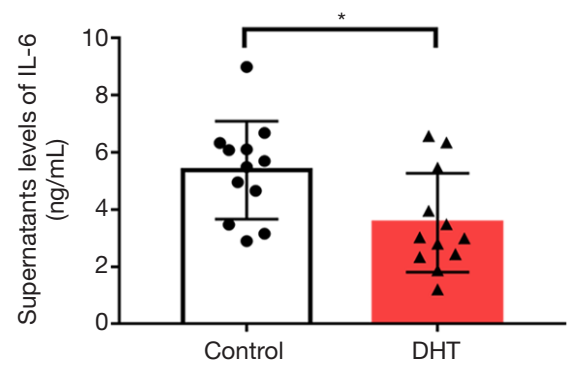

D

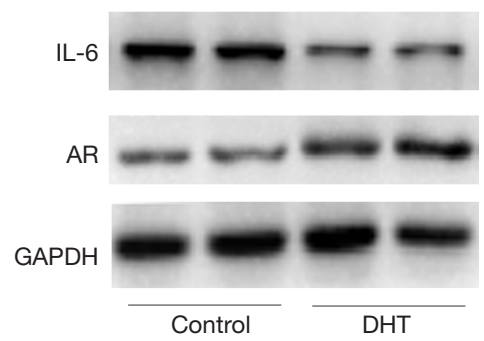

B

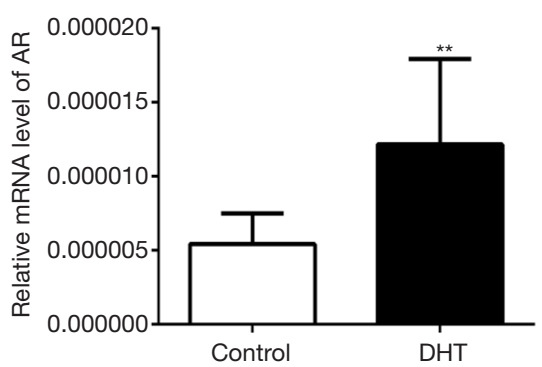

$\mathrm{E}$

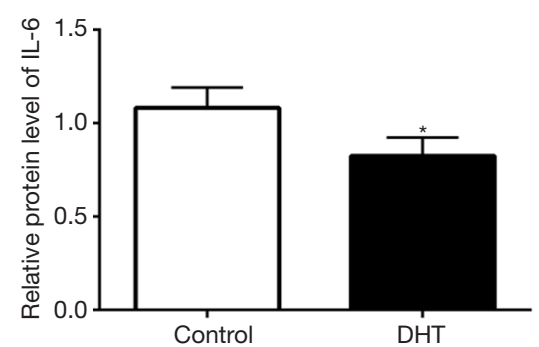

C

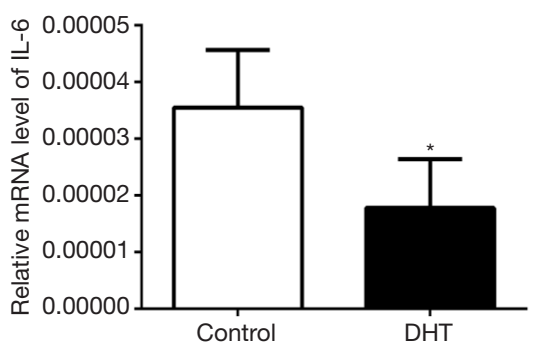

$\mathrm{F}$

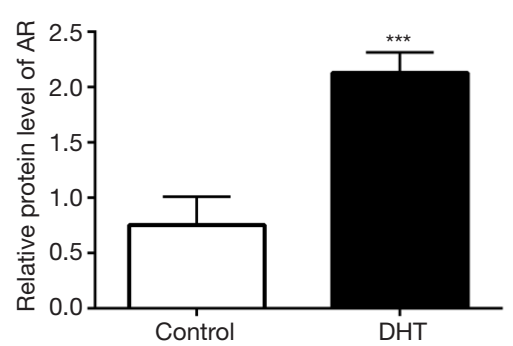

Figure 3 Androgen inhibited the secretion of IL-6 in AIS primary chondrocytes. (A) The IL-6 level in the AIS primary chondrocyte culture medium after DHT treatment. (B,C) The relative mRNA levels of AR and IL-6 in AIS primary chondrocytes after the DHT intervention. $(\mathrm{D}, \mathrm{E}, \mathrm{F})$ The protein levels of AR and IL-6 in AIS primary chondrocytes after DHT treatment. The data are shown as the means \pm SDs. *indicates $\mathrm{P}<0.05 v$ s. the control group.

A

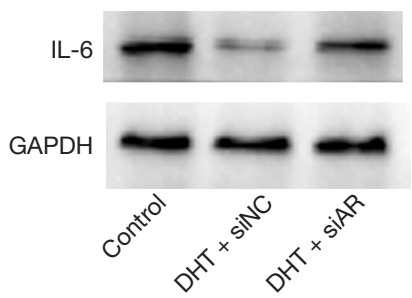

B

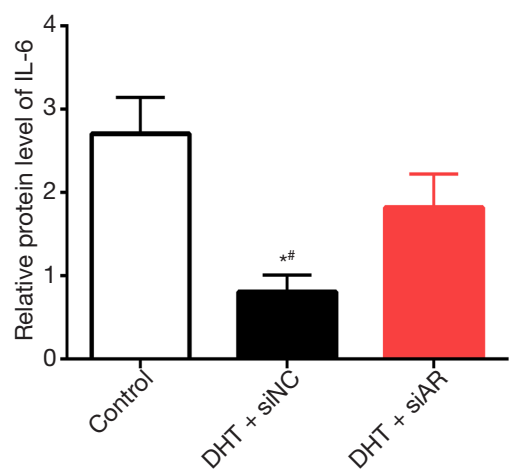

$\mathrm{C}$

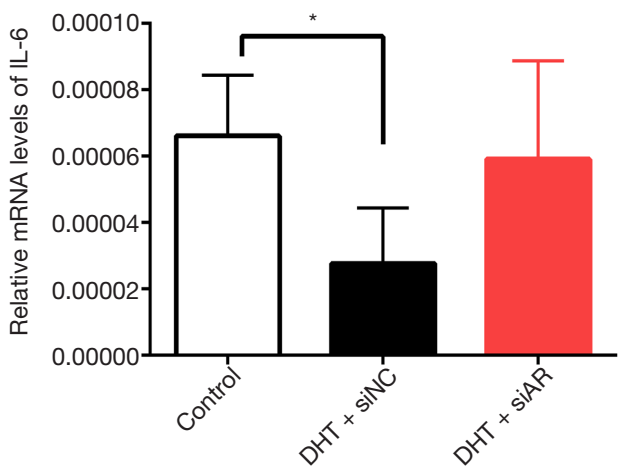

Figure 4 (A,B) The protein level of IL-6 in AIS primary chondrocytes after transfection. (C) The relative mRNA level of IL-6 in AIS primary chondrocytes after transfection. The data are shown as the means \pm SDs. *indicates $\mathrm{P}<0.05$ vs. the control group; ${ }^{*}$ indicates $\mathrm{P}<0.05$ vs. the DHT + siAR group.

from AIS patients was achieved through activation of STAT3 protein, we cultured AIS primary chondrocytes with Stattic (MCE, USA), a nonpeptidic small molecule that specifically inhibits STAT3. The results showed that the level of phosphorylated STAT3 protein increased after treating with $20 \mathrm{nM}$ IL-6 for $48 \mathrm{~h}$, and the expression of the STAT3 downstream protein MMP13 also increased. However, STAT3 phosphorylation was inhibited in the IL-6 + Stattic group, and accordingly, the protein expression of MMP13 was also decreased in this group (Figure 6C,D). These results indicate that the regulatory function of IL-6 on chondrocytes is achieved through STAT3 protein 
A

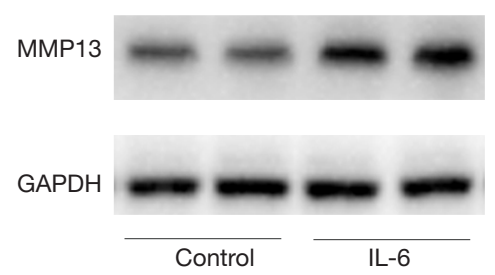

C

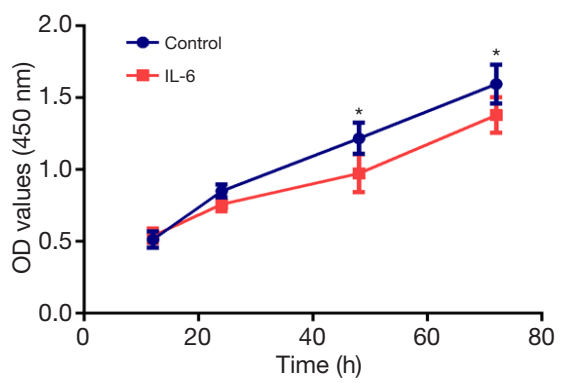

E
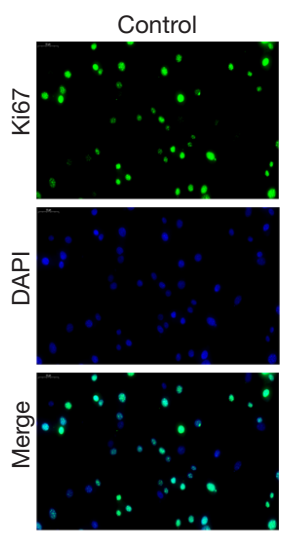
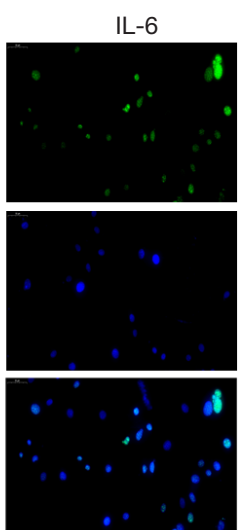

B

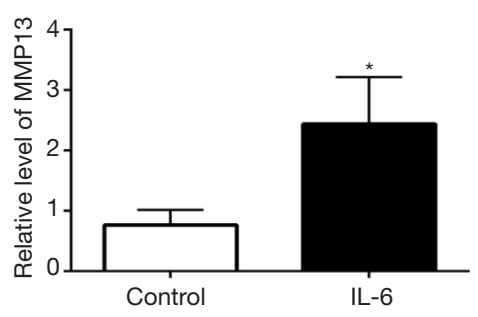

D

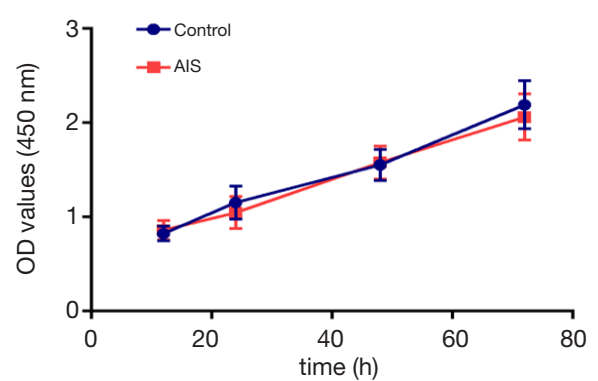

$\mathrm{F}$

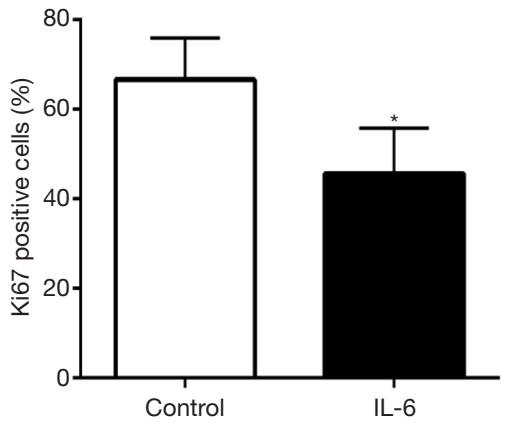

Figure 5 Promotion of MMP13 protein expression and inhibition of chondrocyte proliferation by IL-6. (A,B) The protein level of MMP13 in AIS primary chondrocytes after the intervention. (C) Chondrocyte proliferation was measured 12, 24, 48, and $72 \mathrm{~h}$ after treatment. (D) The proliferation of chondrocytes from AIS patients and non-AIS patients. (E,F) The proliferation of AIS primary chondrocytes after treatment with $20 \mathrm{nM} \mathrm{IL}-6$ for $48 \mathrm{~h}$, green signal, 400x. The data are shown as the means \pm SDs. *indicates $\mathrm{P}<0.05$ vs. the control group.

activation.

\section{Discussion}

AIS causes three-dimensional deformation of the spine. Although its pathogenesis remains unclear, numerous experiments have suggested that AIS development is closely associated with genetic factors $(2,23)$ and hormone secretion $(4,24)$. In addition, previous studies have confirmed that chondrocyte activity on the AIS patients and non-AIS patients is different (25) and that endochondral ossification is a very important process in the human body.
These factors might cause unbalanced growth between the convex and concave sides of the spine, which would eventually result in deformity (26). Therefore, we collected facet joint tissue during surgery for IHC and WB analyses, and the results showed that AR expression and the levels of IL-6, MMP9 and MMP13 were significantly increased in the cartilage tissue of AIS patients, which might be important factors inducing abnormal development or chondrocyte degradation in patients with AIS.

High levels of a number of cellular and soluble factors, including inflammatory cytokines, such as IL-6, are present in AIS cartilage (18). IL-6 causes cartilage damage by acting 
A

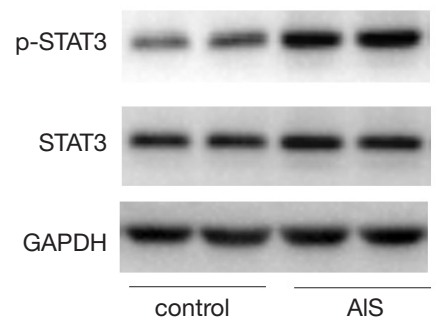

C

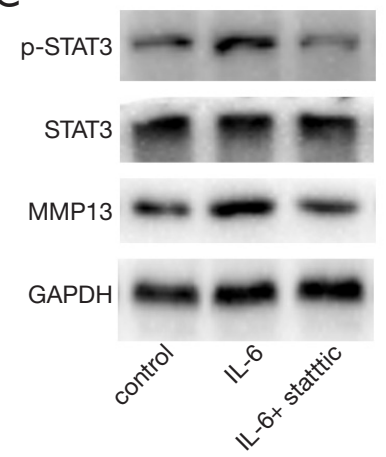

B

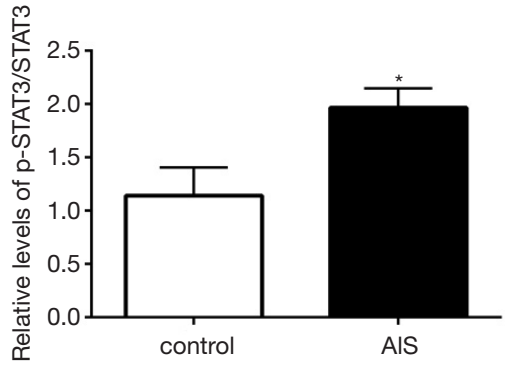

$\mathrm{D}$

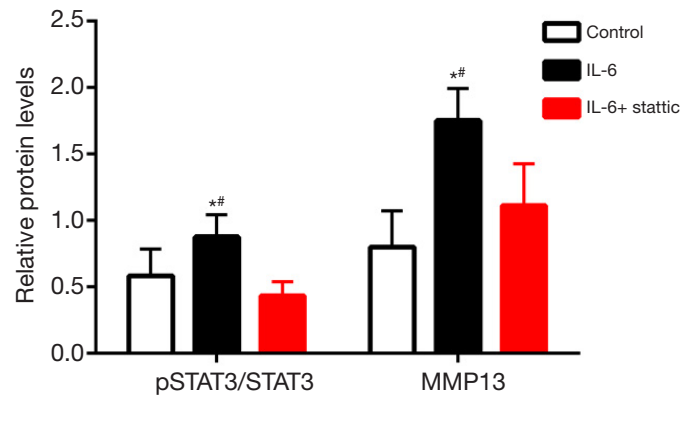

Figure 6 Effects of IL-6 on chondrocytes from AIS patients mediated by STAT3 signaling pathway proteins. Total and phosphor-STAT3 protein levels in AIS and non-AIS patients (A,B). Total, phosphor-STAT3, and MMP13 protein levels in AIS primary chondrocytes after the intervention $(\mathrm{C}, \mathrm{D})$. The data are shown as the means \pm SDs. ${ }^{*}$ indicates $\mathrm{P}<0.05$ vs. the control group; ${ }^{\#}$ indicates $\mathrm{P}<0.05$ vs. the IL- $6+$ Stattic group.

on both anabolic and catabolic mechanisms of cartilage physiology (27). After the initial finding of abnormal IL-6 levels in cartilage tissue, these results were further validated through cell culture experiments. In this research, primary chondrocytes from AIS patients were stimulated with $20 \mathrm{nM}$ IL-6, and the results showed that IL-6 promoted MMP13 protein expression and inhibited chondrocyte proliferation. IL-6 was previously found to be a crucial mediator of the MMP13 levels in chondrocytes (28), and other studies have shown that IL- 6 inhibits the expression of type II collagen (29). The most specific downstream protein in the IL-6 pathway is STAT3, and numerous studies have shown that IL- 6 activates STAT3 protein in chondrocytes to regulate the expression levels of downstream proteins $(22,30,31)$. Our results also showed that IL-6 exerted its function in chondrocytes from patients with AIS through STAT3 protein activation. IL-6 and MMP13 are usually associated with cartilage catabolism, Ryu et al. found that IL-6 induced experimental osteoarthritis cartilage destruction in mice via regulation of MMP13 levels (32). Bouaziz et al. indicated the lack of MMP13 protects the articular cartilage from degradation and osteoarthritis in mice (33). Therefore, the detected increase in IL-6 expression in patients with AIS might induce an inflammatory reaction in chondrocytes to accelerate degradation and thus induced unbalanced vertebral growth.

Previous studies have confirmed that androgen can promote cortical bone formation through IL-6, which indicated that androgen plays a very important regulatory role in bone formation and growth (17). A study conducted by ElBaradie et al. demonstrated that DHT regulates growth plate chondrocytes in a sex-specific manner via rapid signaling pathways (34). In an earlier study, Raz et al. found that rat costochondral growth plate chondrocytes exhibit sex-specific and cell maturation-dependent responses to testosterone. In addition, these researchers proposed that the specific response of chondrocytes to hormones requires further metabolism of testosterone to DHT (35). Therefore, in our study, primary chondrocytes from AIS patients were treated with $5 \times 10^{-7} \mathrm{M}$ DHT, and the results showed that androgen played an important role in cartilage through IL-6. Specifically, DHT inhibited IL-6 expression through AR activation in chondrocytes of AIS patients. In 


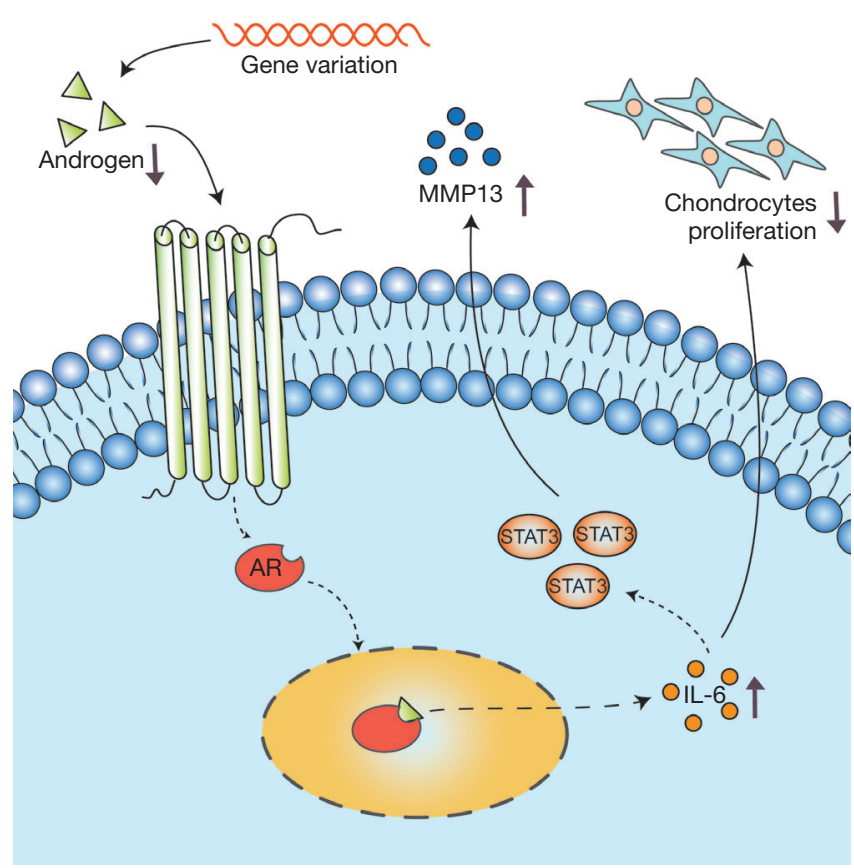

Figure 7 Schematic diagram of androgen in the abnormal development of cartilage in female AIS patients.

addition, the serum androgen levels in patients with AIS were lower than those in the non-AIS group, which resulted in a weakened inhibitory function of androgen on IL-6 in patients with AIS. These results suggest that androgen might play a protective role in the development of AIS to inhibit IL-6-induced abnormal chondrocyte development.

We investigated the reasons for the lower serum androgen levels in patients with AIS. A previous study on the relationship between estrogen and AIS showed that patients with AIS have low serum androgen levels in the body, but no further studies have been conducted (8). In addition, Raczkowski found increased testosterone levels above the reference values in girls with AIS, though that study only included 27 samples in the AIS group and seven samples in the control group, which can easily lead to errors in the experiment (36). Based on studies of the gene sequence of androgen, we speculated that many allelic SNPs might affect the androgen secretion levels. Recent studies confirmed that SNP loci, including rs12150660, rs727428, rs6259, rs5934505, and rs10822184, are associated with androgen levels $(14,15)$. Therefore, 161 females with AIS and 140 females without AIS were recruited for genotyping. The results showed that the mutation rates of rs6259 were significantly different between the AIS and
non-AIS groups, and the G/G and G/A allelic mutation rates were significantly higher in the AIS group (G/G genotype: $48.4 \%$ vs. $42.1 \%$, G/A genotype: $40.4 \%$ vs. $35.7 \%$, and $\mathrm{A} / \mathrm{A}$ genotype: $11.2 \%$ vs. $22.2 \% ; \mathrm{P}<0.05$ ). A study performed by Martínez-García MÁ et al. showed that rs6259 is a nonsynonymous mutation, and this SNP reduces the expression of androgen through an increase in the sex hormone-binding globulin gene (SHBG) (16), which is consistent with our results. Many studies have also revealed that rs6259 can increase the risk of polycystic ovary syndrome (37), diabetes mellitus (38), and metastatic prostate cancer (39), among others, but no research on the relationship between rs6259 and AIS has been reported.

This study also has some limitations. First, we could not confirm whether androgen was the most important factor in AIS development. Androgen might affect abnormal cartilage development in patients with AIS in combination with other hormones or factors. Second, the chondrocytes extracted in this study were all obtained from the cartilage tissue of the facet joint of the spine, which is not representative of the different cartilage tissues throughout the body. Third, the experimental samples included in this study were limited and cannot fully reflect differences in sex, Cobb angle, and race.

In conclusion, this study provides a new perspective for studying the pathogenesis of AIS. Our results show that androgen can reduce abnormal cartilage development in patients with AIS through AR/IL-6/STAT3 pathway inhibition (Figure 7). The data suggest that decreased androgen levels in female AIS patients might play a potential role in the occurrence of AIS.

\section{Acknowledgments}

Funding: This study was supported by the Natural Science Foundation of China (No. 81772298).

\section{Footnote}

Data Sharing Statement: Available at http://dx.doi. org/10.21037/atm-20-3171

Conflicts of Interest: All authors have completed the ICMJE uniform disclosure form (available at http://dx.doi. org/10.21037/atm-20-3171). The authors have no conflicts of interest to declare.

Ethical Statement: All authors are accountable for all 
aspects of the work in ensuring that questions related to the integrity or accuracy of the work are appropriately investigated and resolved. This study was approved by the Ethics Committee of Xiangya Hospital of Central South University (No. 201703358). Written informed consent was provided by all the subjects and their legal guardians prior to participation in the study, and the study was performed in accordance with the tenets of the Declaration of Helsinki (as revised in 2013) and its amendments.

Open Access Statement: This is an Open Access article distributed in accordance with the Creative Commons Attribution-NonCommercial-NoDerivs 4.0 International License (CC BY-NC-ND 4.0), which permits the noncommercial replication and distribution of the article with the strict proviso that no changes or edits are made and the original work is properly cited (including links to both the formal publication through the relevant DOI and the license). See: https://creativecommons.org/licenses/by-nc-nd/4.0/.

\section{References}

1. Cheng JC, Castelein RM, Chu WC, et al. Adolescent idiopathic scoliosis. Nat Rev Dis Primers 2015;1:15030.

2. Haller G, McCall K, Jenkitkasemwong S, et al. A missense variant in SLC39A8 is associated with severe idiopathic scoliosis. Nat Commun 2018;9:4171.

3. Blecher R, Krief S, Galili T, et al. The Proprioceptive System Masterminds Spinal Alignment: Insight into the Mechanism of Scoliosis. Dev Cell 2017;42:388-99.e3.

4. Zheng S, Zhou H, Gao B, et al. Estrogen promotes the onset and development of idiopathic scoliosis via disproportionate endochondral ossification of the anterior and posterior column in a bipedal rat model. Exp Mol Med 2018;50:1-11.

5. Clark EM, Taylor HJ, Harding I, et al. Association between components of body composition and scoliosis: a prospective cohort study reporting differences identifiable before the onset of scoliosis. J Bone Miner Res 2014;29:1729-36.

6. Liang ZT, Li J, Rong R, et al. Ghrelin up-regulates cartilagespecific genes via the ERK/STAT3 pathway in chondrocytes of patients with adolescent idiopathic scoliosis. Biochem Biophys Res Commun 2019;518:259-65.

7. Ueno M, Takaso M, Nakazawa T, et al. A 5-year epidemiological study on the prevalence rate of idiopathic scoliosis in Tokyo: school screening of more than 250,000 children. J Orthop Sci 2011;16:1-6.
8. Esposito T, Uccello R, Caliendo R, et al. Estrogen receptor polymorphism, estrogen content and idiopathic scoliosis in human: a possible genetic linkage. J Steroid Biochem Mol Biol 2009;116:56-60.

9. Dewailly D, Robin G, Peigne $M$, et al. Interactions between androgens, FSH, anti-Mullerian hormone and estradiol during folliculogenesis in the human normal and polycystic ovary. Hum Reprod Update 2016;22:709-24.

10. Almeida $M$, Laurent MR, Dubois V, et al. Estrogens and Androgens in Skeletal Physiology and Pathophysiology. Physiol Rev 2017;97:135-87.

11. Abu EO, Horner A, Kusec V, et al. The localization of androgen receptors in human bone. J Clin Endocrinol Metab 1997;82:3493-7.

12. Cicuttini FM, Wluka A, Bailey M, et al. Factors affecting knee cartilage volume in healthy men. Rheumatology (Oxford) 2003;42:258-62.

13. Steffens JP, Coimbra LS, Rossa C Jr, et al. Androgen receptors and experimental bone loss - an in vivo and in vitro study. Bone 2015;81:683-90.

14. Chen YP, Nie LL, Li HG, et al. The rs5934505 single nucleotide polymorphism (SNP) is associated with low testosterone and late-onset hypogonadism, but the rs10822184 SNP is associated with overweight and obesity in a Chinese Han population: a case-control study. Andrology 2016;4:68-74.

15. Jin G, Sun J, Kim ST, et al. Genome-wide association study identifies a new locus JMJD1C at 10q21 that may influence serum androgen levels in men. Hum Mol Genet 2012;21:5222-8.

16. Martinez-Garcia MA, Gambineri A, Alpanes M, et al. Common variants in the sex hormone-binding globulin gene (SHBG) and polycystic ovary syndrome (PCOS) in Mediterranean women. Hum Reprod 2012;27:3569-76.

17. Cho DC, Brennan HJ, Johnson RW, et al. Bone corticalization requires local SOCS3 activity and is promoted by androgen action via interleukin- 6 . Nat Commun 2017;8:806.

18. Zhang HQ, Wang LJ, Liu SH, et al. Adiponectin regulates bone mass in AIS osteopenia via RANKL/OPG and IL6 pathway. J Transl Med 2019;17:64.

19. Laavola M, Leppanen T, Hamalainen M, et al. IL-6 in Osteoarthritis: Effects of Pine Stilbenoids. Molecules 2018;24:109.

20. Ushiyama $\mathrm{T}$, Chano $\mathrm{T}$, Inoue $\mathrm{K}$, et al. Cytokine production in the infrapatellar fat pad: another source of cytokines in knee synovial fluids. Ann Rheum Dis 2003;62:108-12.

21. Yamaguchi R, Kamiya N, Adapala NS, et al. HIF-1- 
Dependent IL-6 Activation in Articular Chondrocytes Initiating Synovitis in Femoral Head Ischemic Osteonecrosis. J Bone Joint Surg Am 2016;98:1122-31.

22. Kondo M, Yamaoka K, Sakata K, et al. Contribution of the Interleukin-6/STAT-3 Signaling Pathway to Chondrogenic Differentiation of Human Mesenchymal Stem Cells. Arthritis Rheumatol 2015;67:1250-60.

23. Xu JF, Yang GH, Pan XH, et al. Association of GPR126 gene polymorphism with adolescent idiopathic scoliosis in Chinese populations. Genomics 2015;105:101-7.

24. Machida M, Dubousset J, Yamada T, et al. Serum melatonin levels in adolescent idiopathic scoliosis prediction and prevention for curve progression--a prospective study. J Pineal Res 2009;46:344-8.

25. Wang S, Qiu Y, Zhu Z, et al. Histomorphological study of the spinal growth plates from the convex side and the concave side in adolescent idiopathic scoliosis. J Orthop Surg Res 2007;2:19.

26. Bylski-Austrow DI, Glos DL, Wall EJ, et al. Scoliosis vertebral growth plate histomorphometry: Comparisons to controls, growth rates, and compressive stresses. J Orthop Res 2018;36:2450-9.

27. Liu X, Croker BA, Campbell IK, et al. Key role of suppressor of cytokine signaling 3 in regulating gp130 cytokine-induced signaling and limiting chondrocyte responses during murine inflammatory arthritis. Arthritis Rheumatol 2014;66:2391-402.

28. Zanotti S, Canalis E. Interleukin 6 mediates selected effects of Notch in chondrocytes. Osteoarthritis Cartilage 2013;21:1766-73.

29. Nasi S, So A, Combes C, et al. Interleukin-6 and chondrocyte mineralisation act in tandem to promote experimental osteoarthritis. Ann Rheum Dis 2016;75:1372-9.

30. Latourte A, Cherifi C, Maillet J, et al. Systemic inhibition of IL-6/Stat3 signalling protects against experimental osteoarthritis. Ann Rheum Dis 2017;76:748-55.

31. Wiegertjes R, van Caam A, van Beuningen H, et al. TGF-

Cite this article as: Wu YT, Tang MX, Wang YJ, Li J, Wang YX, Deng A, Guo CF, Zhang HQ. Lower androgen levels promote abnormal cartilage development in female patients with adolescent idiopathic scoliosis. Ann Transl Med 2021;9(9):784. doi: 10.21037/atm-20-3171 beta dampens IL-6 signaling in articular chondrocytes by decreasing IL-6 receptor expression. Osteoarthritis Cartilage 2019;27:1197-207.

32. Ryu JH, Yang S, Shin Y, et al. Interleukin-6 plays an essential role in hypoxia-inducible factor 2alpha-induced experimental osteoarthritic cartilage destruction in mice. Arthritis Rheum 2011;63:2732-43.

33. Bouaziz W, Sigaux J, Modrowski D, et al. Interaction of HIF $1 \alpha$ and $\beta$-catenin inhibits matrix metalloproteinase 13 expression and prevents cartilage damage in mice. Proc Natl Acad Sci U S A 2016;113:5453-8.

34. ElBaradie K, Wang Y, Boyan BD, et al. Rapid membrane responses to dihydrotestosterone are sex dependent in growth plate chondrocytes. J Steroid Biochem Mol Biol 2012;132:15-23.

35. Raz P, Nasatzky E, Boyan BD, et al. Sexual dimorphism of growth plate prehypertrophic and hypertrophic chondrocytes in response to testosterone requires metabolism to dihydrotestosterone (DHT) by steroid 5-alpha reductase type 1. J Cell Biochem 2005;95:108-19.

36. Raczkowski JW. The concentrations of testosterone and estradiol in girls with adolescent idiopathic scoliosis. Neuro Endocrinol Lett 2007;28:302-4.

37. Liu Y, Zhao XX, Hu XJ, et al. Effect of sex hormonebinding globulin polymorphisms on the outcome of in vitro fertilization-embryo transfer for polycystic ovary syndrome patients: A case-control study. J Cell Biochem 2019;120:4675-86.

38. Zhang X, Sun L, Jin Z. Effect of placental sex hormonebinding globulin single nucleotide polymorphism rs6259 on protein and function in gestational diabetes mellitus. Int J Mol Med 2018;41:2927-34.

39. Shiota M, Fujimoto N, Tsukahara S, et al. Genetic Polymorphism in Sex Hormone-binding Globulin With a Prognosis of Androgen Deprivation Therapy in Metastatic Prostate Cancer Among Japanese Men. Clin Genitourin Cancer 2019;17:e387-93. 


\section{Supplementary}

Table S1 siRNA sequences used in this study

\begin{tabular}{ll}
\hline SiRNA & Sequences \\
\hline siAR 1 & Sense: 5'-GGCAAGAGCACUGAAGAUAdTdT-3' \\
& Antisense: 5'-UAUCUUCAGUGCUCUUGCCdTdT-3' \\
siAR 2 & Sense: 5'-CUGCUACUCUUCAGCAUUAdTdT-3' \\
& Antisense: 5'-UAAUGCUGAAGAGUAGCAGdTdT-3' \\
siControl & Sense: 5'-UUCUCCGAACGUGUCACGUdTdT-3' \\
& Antisense: 5'-ACGUGACACGUUCGGAGAAdTdT-3' \\
\hline
\end{tabular}
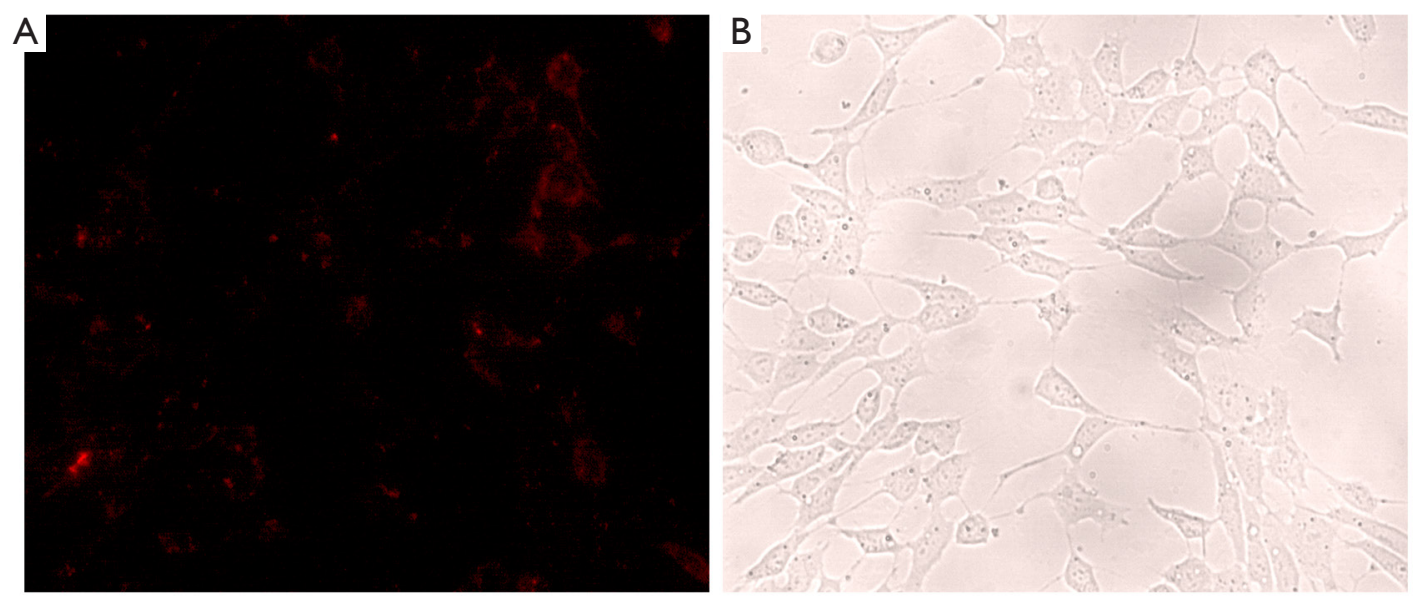

Figure S1 Primary chondrocytes of AIS patients were transfected with the siRNA. (A) AR was labeled with red fluorescence, 400×, strong signals of AR were observed in the cells transfected (B).

A

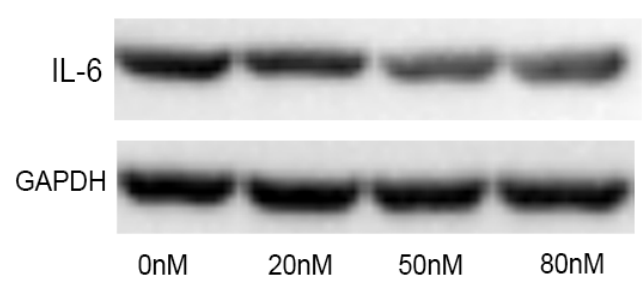

B

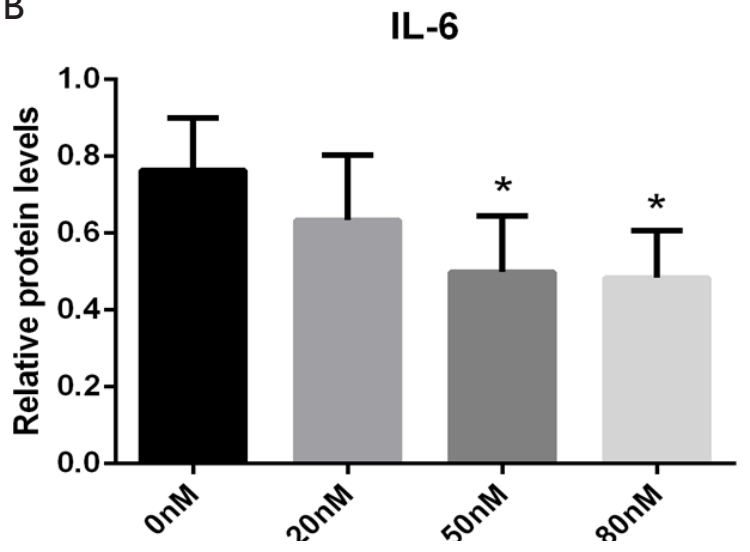

Figure S2 The effect of androgen intervention is effective when the concentration is $50 \mathrm{nM} / 80 \mathrm{nM}$. (A,B) the expression of IL-6 after treating with gradient concentration of androgen. *indicates $\mathrm{P}<0.05 v$ s. the control group. 
A

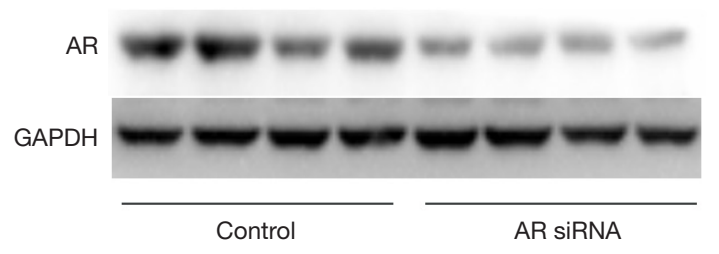

B

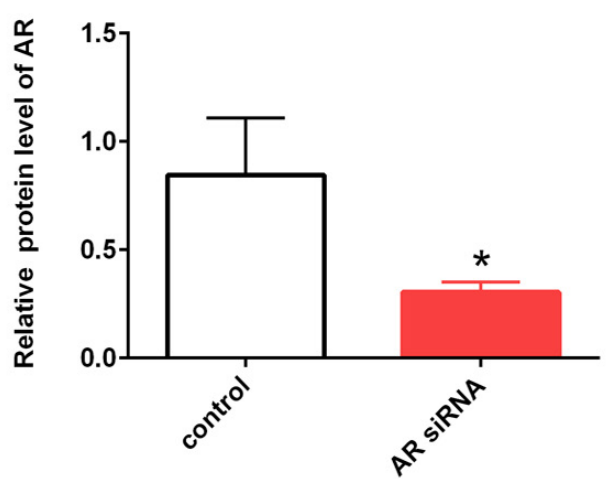

Figure S3 $(\mathrm{A}, \mathrm{B})$ The expression level of AR protein in AIS primary chondrocytes $48 \mathrm{~h}$ after transfection. *indicates $\mathrm{P}<0.05 v s$. the control group.

A

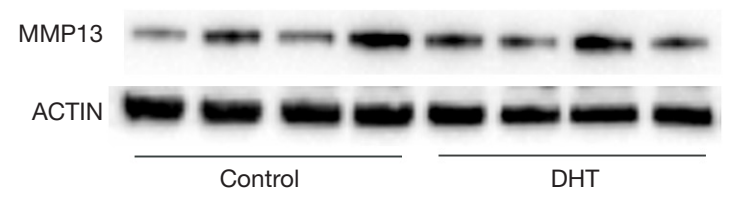

B

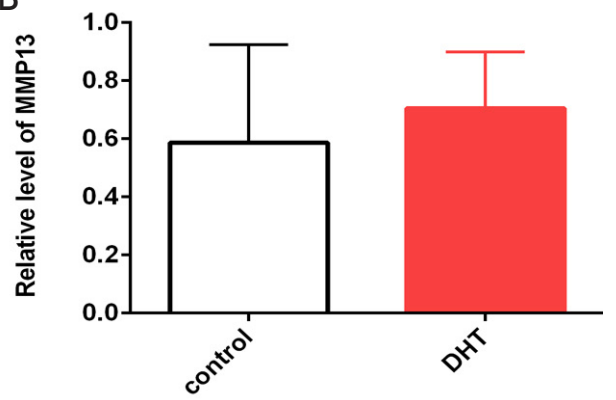

Figure S4 The expression of MMP13 did not significantly change after androgen treatment. (A,B) the protein level of MMP13 in chondrocytes from AIS patients after DHT treatment. *indicates $\mathrm{P}<0.05 v$ s. the control group.

A
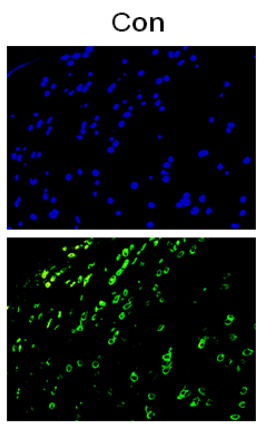

Ki67

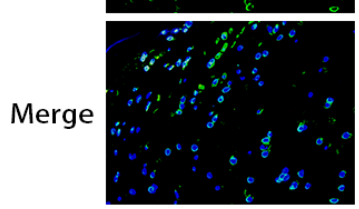

AIS
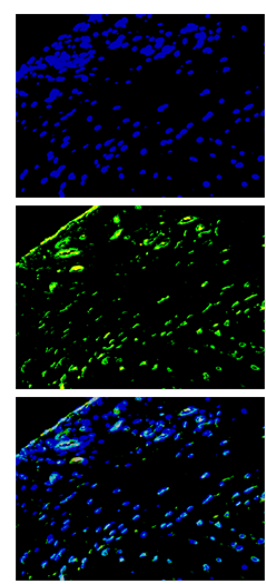

B

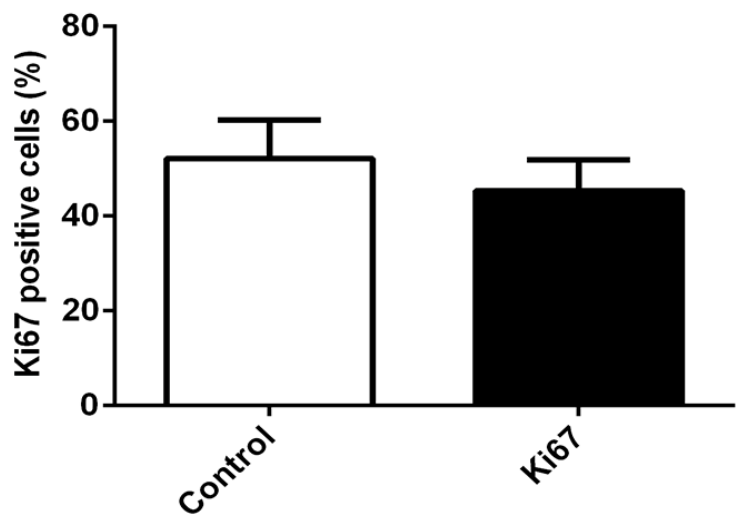

Figure S5 The proliferation of chondrocytes in cartilage from AIS and non-AIS patients. (A,B) There was no significant difference between two groups (green signal), 400x. 